\title{
Septum of the gallbladder, clinical implications and treatment
}

\author{
Alexander A. Deutsch, Dov Englestein, Maya Cohen, M. Kunichevsky and Raphael \\ Reiss
}

Tel Aviv University Medical School, Tel Aviv, Israel

\begin{abstract}
Summary: We report four patients with a congenital gallbladder septum whose symptoms resembled those of cholelithiasis, in one case giving rise to acute cholecystitis. Cholecystectomy relieved symptoms in all cases and examination of the operative specimen confirmed the clinical diagnosis and $\mathrm{X}$-ray findings. Ultrasonography made a positive diagnosis in the last two cases and no stones were found in any of the cases described. Cholecystectomy is advocated in symptomatic patients with this condition, even when gallstones are not present.
\end{abstract}

\section{Introduction}

A septum which divides the gallbladder into two cavities joined by a canal has been variously described as a stricture (Beilby, 1967), a septate gallbladder (Mohan, 1965), and an 'hourglass' gallbladder (Harlafit et al., 1977). A careful study by Beilby (1967) indicates that one to three septae may not be uncommon. This is in contrast to multiple septae where only 13 authenticated cases have been documented (Jena et al., 1977; Toombs et al., 1982). A differentiation can be made between congenital and acquired forms of septum by the presence of adenomyomatosis in the acquired group (Jutras et al., 1960). However, not all authors agree (Aguirre et al., 1969). Adenomyomatosis may be of a segmental nature, also giving rise to an 'hourglass' deformity of the gallbladder.

The phrygian cap, a triangular deformity of the gallbladder fundus as seen on X-ray and ultrasound, is a fairly common anatomical variant. It probably has a similar aetiology to a congenital septum, although it does not give rise to symptoms, as the gallbladder lumen is not narrowed. Spech et al. (1975) found a transverse septum in $4 \%$ of patients having an oral cholecystography and Blumberg (1981) found five cases in 150 cholecystectomy specimens.

Two cases with congenital septum of the gallbladder were previously described (Deutsch et al., 1981) to illustrate a small group of patients who do not have gallstones, but complain of symptoms suggestive of gallbladder pathology. In this group of patients, a

Correspondence: A.A. Deutsch, M.B.Ch.B., F.R.C.S. (England), F.R.C.S. (Edinburgh), Dept. of Surgery B, Beilinson Medical Center, Petah Tiqva 49100, Israel

Accepted: 23 December 1985 diagnosis of this abnormality and cholecystectomy will relieve them of their symptoms.

We describe a further two patients in whom a preoperative diagnosis was made by an ultrasound examination. The importance of being aware of this condition is stressed.

\section{Case reports}

Case 1

A 30 year old woman complained of a 2-year history of episodes resembling biliary colic of increasing frequency. An oral cholecystogram showed a transverse septum which an ultra-sound examination demonstrated (Figure 1). There were no gallstones. The common bile duct was $4 \mathrm{~mm}$.

At operation, the gallbladder looked normal with no signs of inflammation; there were no stones. Pathological examination showed the gallbladder to be divided into two by a septum, $0.3 \mathrm{~mm}$ in thickness (Figure 2). The fundus area was $0.4 \mathrm{~mm}$ in thickness compared to the proximal part which was only $0.2 \mathrm{~mm}$ thick. On histology there seemed to be some glandular hyperplasia at the fundus area. The post-operative course was uneventful and all her symptoms were relieved. The patient remains well and asymptomatic 18 months after surgery.

\section{Case 2}

A 58 year old man was admitted as an emergency with a clinical picture of acute cholecystitis. An ultrasound examination was carried out which showed a disten- 


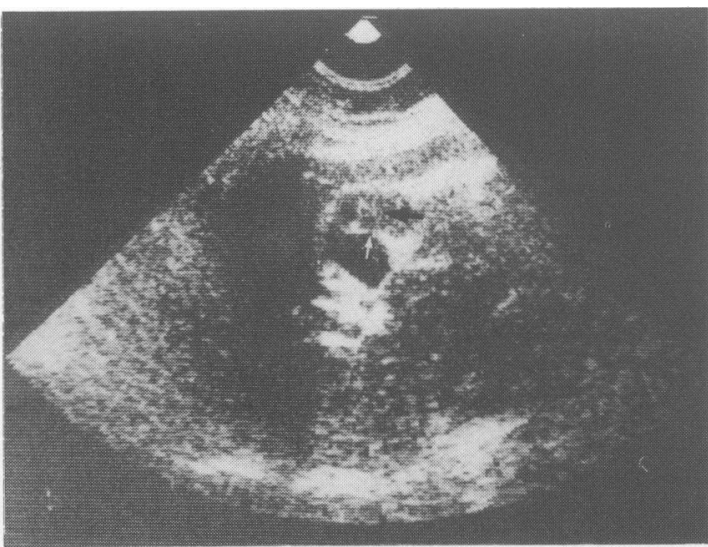

Figure 1 Ultrasound examination: longitudinal section of the right upper abdomen (performed with a $3.5 \mathrm{MHz}$ sector scanner). A normal size gallbladder, divided by a transverse septum (small arrow) into two cavities. A small amount of sludge is shown in the distal cavity (large arrow).

ded gallbladder with a thickened wall. There was also a transverse septum which divided the gallbladder into two cavities (Figure 3). No calculi were seen, and the common bile duct was within normal limits.

At surgery there was acute cholecystitis with a small posterior perforation and intrahepatic abscess formation. Peroperative cholangiography was normal. The post-operative course was uneventful. Pathological examination of the specimen showed severe cholecystitis, particularly of the fundus area, with a septum which divided the gallbladder into two; there were no stones (Figure 4).

Histological examination of the septal area showed that the septum consisted of all layers of the gallbladder with severe inflammation of the fundus side. The patient is asymptomatic a year after operation.

\section{Case 3}

A 30 year old male was admitted with a 4-year history of recurrent episodes of biliary colic. An oral cholecystogram revealed two areas of opacification of the gallbladder divided by a septum. At cholecystectomy, adhesions around the gallbladder were found and when the septum was opened, two distinct cavities, joined by a narrow canal, were seen. There were no gallstones. Histology showed a septum of normal mucosa and muscularis with inflammation. [This case was previously reported by Deutsch et al., 1981).

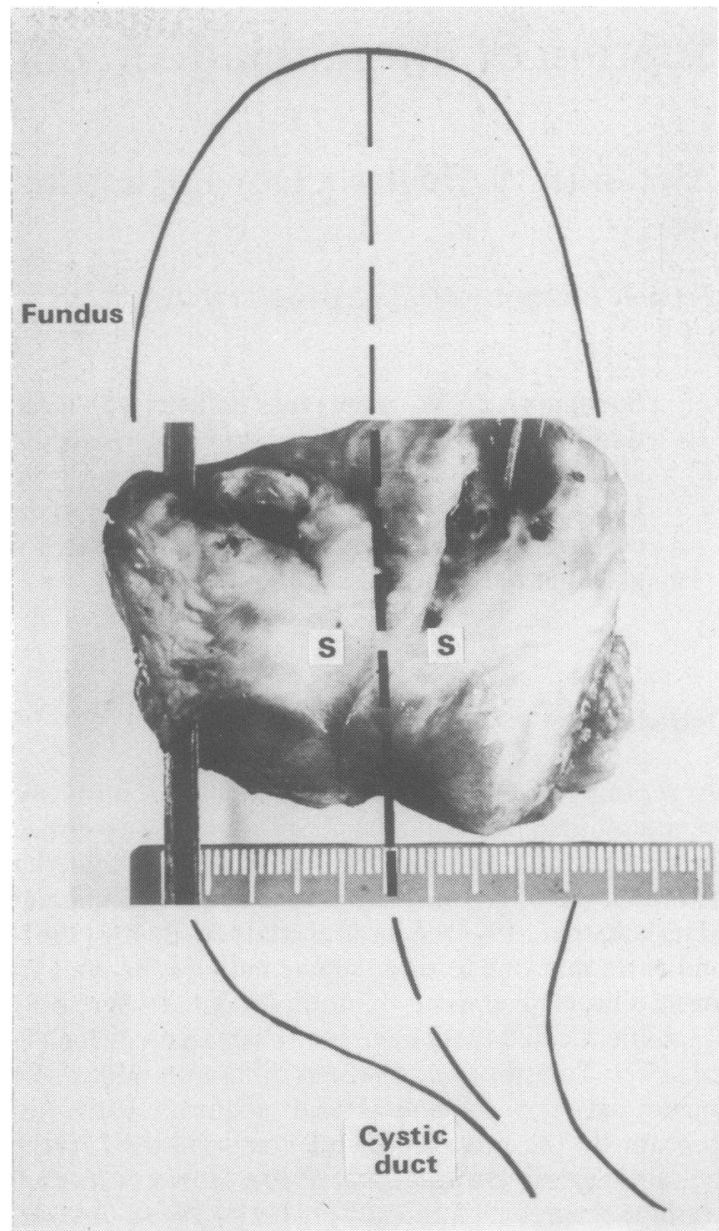

Figure 2 Longitudinal section of gallbladder showing a septum (S) in the middle of the body dividing the lumen in two parts. The cannula passed through the lumen. Stones were not present.

\section{Case 4}

A 37 year old female was admitted with a 12-year history of recurrent pain in the right hypochondrium. An intravenous cholangiography showed two gallbladder cavities with a transverse septum.

At cholecystectomy a diagnosis of septate gallbladder was confirmed. A narrow communication was seen between the two cavities, and no stones were found. Histological examination showed changes of chronic inflammation, with no evidence of muscular hypertrophy in the distal cavity or of adenomyomatosis. (This case was previously reported by Deutsch et al., 1981.) 


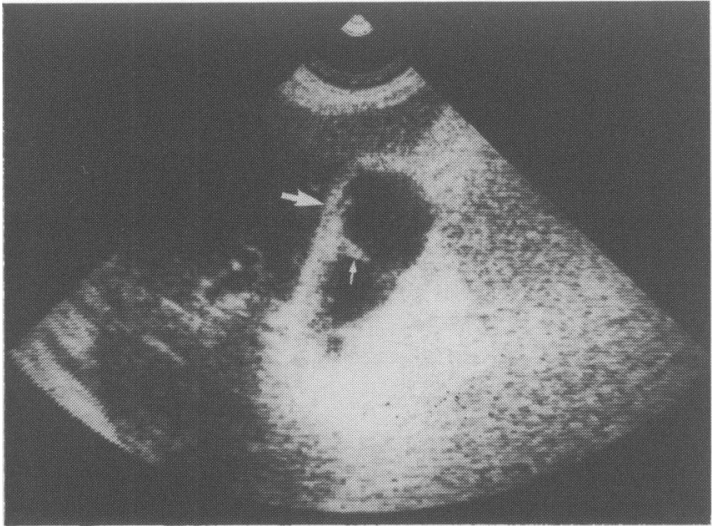

Figure 3 Ultrasound examination: longitudinal section of the right upper abdomen. The gallbladder is distended, with a thickened wall (large arrow). A transverse septum (small arrow) divides the gallbladder into two cavities.

\section{Discussion}

There are few published reports of septate gallbladders although it is generally stated that they are not uncommon at necropsy and in surgical specimens (Aguirre et al., 1969). Beilby (1967) described 52 gallbladder strictures of all types from a variety of surgical and post-mortem material. He describes the occurrence of cholecystitis glandularis proliferans (syn. adenomyomatosis) in the distal cavity in most cases and points out that only 21 of the specimens had strictures less than $12 \mathrm{~mm}$ in diameter. Only one of all specimens described in the above series was completely calculus-free as in our own cases. Hatfield \& Wise (1976) state that congenital transverse septa are not uncommon and are usually of no consequence clinically. Spech et al. (1975) found an incidence of $4 \%$ of all oral cholecystograms and Blumberg (1981) found five cases in 150 cholecystectomy specimens. Jutras et al. (1960) made a clear distinction between acquired and congenital forms of septum; the latter should be less than $2 \mathrm{~mm}$ in thickness. The acquired septum occurs as a result of adenomyomatosis of the gallbladder when segmental in nature. Rokitansky Aschoff sinuses were present in gallbladders with an acquired septum although this was not always visible on X-ray. Aguirre et al. (1969) suggest that adenomyomatosis may also occur as a result of a congenital septum. Both our cases 1 and 2 were found to have adenomyomatosis, but we still feel that they are congenital in origin. They both had a thin septum, covered by epithelium on both surfaces, with no deformation of the gallbladder (Blumberg, 1981). In addition, no stones were found in any of the specimens which would have suggested an aquired origin.

The various case reports of multiseptate gallbladders stress the symptomatology which can occur in this condition, and note the importance of cholecystectomy as a means of relieving the patient of his symptoms (Jena et al., 1977). Jutras \& Levesque (1966) have shown that adenomyomatosis, even if not of a segmental nature, may give rise to biliary symptoms. Also cholecystectomy in these patients is frequently followed by a suppression of symptoms, even in the absence of gallstones. The septum, when adenomyomatosis is absent, occurs as a congenital malformation as illustrated by the occurrence of the condition in neonates (Beilby, 1967); adenomyomatosis is not found in this age group, but it seems that it may occur as a secondary phenomenon in congenital septa as in our cases. Various authors have shown how the development is likely to have occurred on an embryological basis (Bhagavan et al., 1970). This being the case, it is probable that the diagnostic problem will arise in a lower age group, and even in children (Haslam et al., 1966).

A phrygian cap is a common variant, and does not give rise to symptoms, although it may be considered a non-developed form of congenital septum. In order to cause symptoms the connection between the two gallbladder cavities must be very narrow, obstructing the flow of bile between them. Where a case suggestive of cholelithiasis is encountered, the cholecystogram should be examined very carefully even if no stones are 
seen and repeat examinations performed if symptoms persist. It would appear that it is now possible to demonstrate the gallbladder septum by means of an ultrasonic examination, which will make the diagnosis simpler. This aspect has been reported only once previously (Sansot et al., 1983). In Case 2, a diagnosis was made in the presence of acalculous cholecystitis.

Clearly one would not suggest surgery in every gallbladder showing some anatomical abnormality, but when symptoms are severe in association with the deformity, a laparotomy and cholecystectomy are indicated even if no gallstones are diagnosed.

It is possible that the use of cholecystokinin in

\section{References}

AGUIRRE, J.A., BOHLER, R.O. \& GURAIEB, S. (1969). Hyperplastic cholecystoses: a new contribution to the unitarian theory. American Journal of Roentgenology, Radium Therapy and Nuclear Medicine, 107, 1.

BEILBY, J. (1967). Stricture of the gallbladder. Journal of Pathology, 93, 175.

BHAGAVAN, B.S., AMIN, P.B., LAND, A.S. \& WEINBERG, T.T. (1970). Multiseptate gallbladder embryogenic hypothesis. Archives of Pathology, 89, 382.

BLUMBERG, M. (1981). Transverse septum of the gallbladder. South African Journal of Surgery, 19, 139.

DEUTSCH, A.A., ZAGER, M. \& REISS, R. (1981). Septum of the gallbladder. Harefuah, 100, 225.

HARLAFTIS, N., GRAY, S.W. \& SKANDALAKIS, J.E. (1977). Multiple gallbladders. Surgery, Gynecology \& Obstetrics, $145,928$.

HASLAM, H.A., GAYLOR, B.W. \& EBERT, P.A. (1966). Multiseptate gallbladder. American Journal of Diseases of Children, 112, 600.

HATFIELD, P.M. \& WISE, R.E. (1976). Anatomic variations of gallbladder and bile ducts. Seminars in Roentgenology, 11, 157. reproducing symptoms may help to make a positive pre-operative diagnosis. In Case 4 cholecystokinin did not produce symptoms, but it did underline the difference in degrees of opacification in the two cavities on oral cholecystograms.

There is obvious difficulty in deciding to operate on a patient's gallbladder where stones are not found. There are probably only a few cases where patients should undergo an exploratory laparotomy and cholecystectomy even without stones being present, but surgery should not be withheld from this small group of patients to relieve them of their symptoms.

JENA, P.K., HARDIE, R.A. \& HOBSLEY, M. (1977). Multiseptate hypoplastic gallbladder. British Journal of Surgery, 64, 192.

JUTRAS, J.A., LONGTIN, J.M. \& LEVESQUE, H.P. (1960). Hyperplastic cholecystoses: Hickey lecture. American Journal of Roentgenolology, Radium Therapy \& Nuclear Medicine, 83, 795.

JUTRAS, J.A. \& LEVESQUE, H.P. (1966). Adenomyoma and adenomyomatosis of gallbladder: radiologic and pathologic corrections. Radiologic Clinics of North America, 4, 483.

MOHAN, B. (1965). Septate gallbladder. Canadian Journal of Surgery, 8, 84.

SANSOT, M., VADROT, D., RITANO, D. \& JOUVE, P. (1983) Sonographic images of the 'partitioned' gallbladder. Journal de Radiologie, 64, 687.

SPECH, A., HUMMER, R. \& BROWN, H. (1975). Septierte gallenblussen. Medizinische Klinik, 70, 941.

TOOMBS, B.D., FOUCAR, E., ROWLANDS, B.J. \& STRAY, R. (1982). Multiseptate gallbladder. Southern Medical Journal, 75, 610. 